\title{
Orbifold Reduction Of The Quark-Lepton Symmetric Model
}

\author{
K. L. McDonald and B. H. J. McKellat \\ School of Physics, Research Centre for High Energy Physics, \\ The University of Melbourne, Victoria, 3010, Australia
}

(Dated: April 21, 2018)

\begin{abstract}
We investigate the quark-lepton symmetric gauge group in five dimensions, with the gauge symmetry broken by a combination of orbifold compactification of the extra dimension and the Higgs mechanism. The gauge sector of the model is investigated and contrasted with the four dimensional case. We obtain lower bounds on the mass of the exotic gauge bosons, the inverse compactification scale and the exotic leptons. Light neutrinos are obtained without requiring any scale larger than a TeV. However an ultra-violet cut-off of order $10^{11} \mathrm{GeV}$ is required to suppress proton decay inducing non-renormalizable operators.

PACS numbers: 11.10.Kk, 11.15.Ex, 14.60.Pq, 14.60.St, 14.70.Pw
\end{abstract}

\section{INTRODUCTION}

Quark-lepton (QL) symmetric models, by definition, possess a discrete symmetry whereby one interchanges quarks and leptons in the Lagrangian. Observed low energy phenomena indicate three important differences between quarks and leptons which must be overcome if one is to define such a symmetry. Namely:

i) Quarks display a threefold degeneracy corresponding to the colour degree of freedom — leptons possess no such degeneracy.

ii) The masses of quarks and leptons are distinct and in particular no relationships of the type $m_{e}=m_{u}$ or $m_{e}=m_{d}$ are observed.

iii) Quarks possess fractional electric charges, whilst leptons are integrally charged.

The standard model (SM) of particle physics has demonstrated that the absence of obvious symmetry in low energy phenomena need not imply a corresponding absence in the underlying theory. Spontaneous symmetry breaking has provided a means by which one may construct theories possessing symmetries which are not manifest in the low energy regime.

The construction of a QL symmetric model requires one to attribute the low energy differences between quarks and leptons to the symmetry breaking mechanisms employed in the more symmetric underlying theory. Thus in a QL symmetric theory leptons are expected to come in three leptonic colours, analogous to the three colours of quarks. The symmetry breaking of leptonic colour must then ensure that only one of the three lepton colours remains massless until electroweak symmetry breaking occurs. The different masses and electric charges of quarks and leptons should also arise through the symmetry breaking mechanism.

Previous works [1, 2, 3, 4, 5, 6] have shown that it is possible to construct models which overcome the differences between quarks and leptons listed above, thereby allowing na-

*Electronic address: k.mcdonald@physics.unimelb.edu.au

†Electronic address: b.mckellar@ physics.unimelb.edu.au ture to realize a discrete QL symmetry at high energies. Subsequent symmetry breaking as the universe cools results in a low energy theory indistinguishable from the SM. For other recent works containing leptonic colour see [7, 8, 9, 10, 11].

Studies of QL symmetric models performed to date have employed the Higgs mechanism to break the high energy theory down to the SM. The study of compactified extra dimensions with appropriate boundary conditions has revealed alternative symmetry breaking mechanisms $[12,13,14,15,16$, 17]. Recent years have seen the development of new ideas in symmetry reduction which utilise the discrete symmetry transformations of fields which propagate in compact extra dimensions [18]. These methods have been applied to $S U(5)$ and $S O(10)$ Grand Unified Theories [19, 20, 21, 22, 23, 24. 25, 26, 27, 28, 29, 30, 31, 32, 33, 34, 35, 36, 37, 38], leftright symmetric models [39, 40, 41], the 3-3-1 model [42], unified $S U_{W}(3)$ models [43, 44, 45, 46, 47] and trinification models [48, 49].

In the present work we study a five dimensional QL symmetric model. The QL symmetric gauge group is broken by a combination of orbifold compactification, with the extra dimension forming an $S^{1} / Z_{2} \times Z_{2}^{\prime}$ orbifold, and the usual Higgs mechanism. We assume that fermions are confined to a brane at an orbifold fixed point, whilst scalar and gauge fields propagate in the bulk. The physical spectrum of the resulting model is compared with that of the usual four dimensional models.

We find that the massive gauge bosons associated with the breaking of leptonic colour $S U_{l}(3) \rightarrow S U_{l}(2)$ appear at two scales. The charge $\pm 1 / 2$ exotic $Y$ bosons do not possess zero Kaluza-Klein modes and thus appear only at the inverse compactification scale. Direct bounds on processes mediated by these bosons give $1 / R \geq 5 \mathrm{TeV}$. However the ultraviolet (UV) cut-off of the model is required to be larger than $10^{11} \mathrm{GeV}$ to prevent rapid proton decay. If we assume that the more fundamental theory becomes important just beyond the inverse compactification scale we obtain $1 / R \gtrsim 10^{9} \mathrm{GeV}$ and the $Y$ bosons disappear from the low energy spectrum. An additional neutral gauge boson, $Z^{\prime}$, and exotic charge $1 / 2$ coloured leptons appear at a symmetry breaking scale between the inverse compactification scale and the electroweak scale. This intermediate scale may be as low as a $\mathrm{TeV}$, rendering these states observable at future colliders. This differs from 
four dimensional models where the $Y$ and $Z^{\prime}$ mass scales are coupled.

The layout of this paper is as follows. In Section 1 we review the QL symmetric theory. We develop the symmetry breaking used in the five dimensional theory in Section IIII and study the spectrum of exotic gauge bosons and leptons in Section[IV] In Section[V] we discuss neutrino mass and proton decay within the model and we conclude in Section $\nabla]$

\section{REVIEW OF THE MINIMAL QUARK-LEPTON SYMMETRIC MODEL}

QL symmetry is implemented by extending the SM gauge group to $\mathcal{G}_{q l}=S U_{l}(3) \otimes S U_{q}(3) \otimes S U_{L}(2) \otimes U_{X}(1)$, where $S U_{q}(3)$ is the normal colour group and $X \neq Y$, where $Y$ is the usual SM hypercharge. The matter fields are assigned to the following representations of $\mathcal{G}_{q l}$ :

$$
\begin{aligned}
Q_{L} & \sim(1,3,2,1 / 3), \\
u_{R} & \sim(1,3,1,4 / 3), \quad d_{R} \sim(1,3,1,-2 / 3), \\
F_{L} & \sim(3,1,2,-1 / 3), \\
E_{R} & \sim(3,1,1,-4 / 3), \quad N_{R} \sim(3,1,1,2 / 3),
\end{aligned}
$$

where $F_{L}, N_{R}$ and $E_{R}$ contain the usual left-chiral lepton $S U_{L}(2)$ doublet, right-chiral neutrino and right-chiral charged lepton respectively. The simplest Higgs sector is given by [4]

$$
\begin{aligned}
& \chi \sim(3,1,1,2 / 3), \quad \chi^{\prime} \sim(1,3,1,-2 / 3), \\
& \phi_{1} \sim(1,1,2,1), \quad \phi_{2} \sim(1,1,2,-1) .
\end{aligned}
$$

The discrete QL symmetry acts on the matter and scalar fields as follows:

$$
\begin{aligned}
& Q_{L} \leftrightarrow F_{L}, \quad u_{R} \leftrightarrow E_{R}, \quad d_{R} \leftrightarrow N_{R}, \\
& \chi \leftrightarrow \chi^{\prime}, \quad \phi_{1} \leftrightarrow \phi_{2}, \quad \phi_{1}^{c} \leftrightarrow \phi_{2}^{c},
\end{aligned}
$$

where $\phi_{1,2}^{c} \equiv i \tau_{2} \phi_{1,2}^{*}$. The gauge fields transform as:

$$
G_{q}^{\mu} \leftrightarrow G_{l}^{\mu}, \quad W^{\mu} \leftrightarrow W^{\mu}, \quad C^{\mu} \leftrightarrow-C^{\mu},
$$

where $G_{q, l}^{\mu}$ are the $S U_{q, l}(3)$ gauge bosons, $W^{\mu}$ are the weak bosons and $C^{\mu}$ is the $U_{X}(1)$ gauge boson. The Yukawa Lagrangian may be separated into an electroweak portion,

$$
\begin{aligned}
\mathcal{L}_{e w}= & \lambda_{1}\left(\bar{Q}_{L} d_{R} \phi_{1}+\bar{F}_{L} N_{R} \phi_{2}\right)+\lambda_{1}^{\prime}\left(\bar{Q}_{L} d_{R} \phi_{2}^{c}+\bar{F}_{L} N_{R} \phi_{1}^{c}\right)+\lambda_{2}\left(\bar{Q}_{L} u_{R} \phi_{1}^{c}+\bar{F}_{L} E_{R} \phi_{2}^{c}\right) \\
& +\lambda_{2}^{\prime}\left(\bar{Q}_{L} u_{R} \phi_{2}+\bar{F}_{L} E_{R} \phi_{1}\right)+\text { H.c. }
\end{aligned}
$$

and a non-electroweak portion,

$$
\begin{aligned}
\mathcal{L}_{\text {non-ew }}= & h_{1}\left[\overline{\left(F_{L}\right)^{c}} F_{L} \chi+\overline{\left(Q_{L}\right)^{c}} Q_{L} \chi^{\prime}\right]+ \\
& h_{2}\left[\overline{\left(E_{R}\right)^{c}} N_{R} \chi+\overline{\left(u_{R}\right)^{c}} d_{R} \chi^{\prime}\right]+\text { H.c. },
\end{aligned}
$$

where $\lambda_{1,2}, \lambda_{1,2}^{\prime}$ and $h_{1,2}$ are Yukawa coupling constants. The scalar potential admits a minimum corresponding to [1]

$$
\begin{aligned}
& \left\langle\phi_{1}\right\rangle=\left(0, u_{1}\right)^{T}, \quad\left\langle\phi_{2}\right\rangle=\left(u_{2}, 0\right)^{T}, \\
& \langle\chi\rangle=(w, 0,0)^{T}, \quad\left\langle\chi^{\prime}\right\rangle=0,
\end{aligned}
$$

where an $S U_{l}(3)$ rotation has been performed to obtain the most general vacuum expectation value (VEV) for $\chi$. The vanishing VEV for $\chi^{\prime}$ ensures that $S U_{q}(3)$ remains unbroken whilst the non-zero VEV for $\chi$ breaks the gauge group as

$$
\mathcal{G}_{q l} \rightarrow S U_{l}(2) \otimes S U_{q}(3) \otimes S U_{L}(2) \otimes U_{Y}(1) .
$$

Here the SM hypercharge generator is given by:

$$
Y=X+\frac{1}{\sqrt{3}} T_{8}
$$

where $T_{8}=(1 / \sqrt{3}) \times \operatorname{diag}(-2,1,1)$ is a diagonal generator of $S U_{l}(3)$. At the next stage of symmetry breaking the SM gauge group is broken by the non-zero VEV's for $\phi_{1,2}$.

$$
S U_{l}(2) \otimes \mathcal{G}_{S M} \rightarrow S U_{l}(2) \otimes S U_{q}(3) \otimes U_{Q}(1),
$$

where $\mathcal{G}_{S M}$ is the SM gauge group and $U_{Q}(1)$ is the electromagnetic gauge group. Note that an $S U_{l}(2)$ subgroup of $S U_{l}(3)$ remains unbroken.

At the first stage of symmetry breaking the non-zero VEV for $\chi$ breaks five generators of $\mathcal{G}_{q l}$ and thus five gauge bosons develop masses of order $w$. This amounts to four gauge bosons with charge 1/2 and one neutral gauge boson (which mixes of course with the other neutral gauge bosons). The additional lepton degrees of freedom form vectorial representations of the unbroken subgroup and also acquire masses of order $w$. All SM fermions remain massless at this stage, forming chiral representations of the remaining symmetry. At the second stage of symmetry breaking the SM gauge group is broken by the non-zero VEV's $u_{1,2}$ and the SM fermions acquire Dirac mass terms. The electroweak Yukawa Lagrangian (5) ensures that all SM fermions may have unique masses with no relations of the type $m_{e}=m_{u}$ or $m_{e}=m_{d}$ arising (the field $\phi_{2}$ is included for this very purpose). The photon remains massless whilst the other SM gauge bosons develop order $u_{1,2}$ masses.

Thus, to surmise, the model predicts an additional massive neutral gauge boson $Z^{\prime}$ and four charge $1 / 2$ massive gauge bosons, which we generically label as $Y$, all with order $w$ masses. It also predicts additional leptons, forming non-trivial representations of the unbroken $S U_{l}(2)$, with order $w$ masses. 
Phenomenology of these additional states can be found in [3].

\section{ORBIFOLD REDUCTION OF QUARK-LEPTON SYMMETRY}

Having reviewed the standard four dimensional QL symmetric model we now develop a five dimensional version of the model. The additional spatial dimension is taken as the orbifold $S^{1} / Z_{2} \times Z_{2}^{\prime}$, whose coordinate is labelled as $y$. The construction of the orbifold proceeds via the identification $y \rightarrow-y$ under the $Z_{2}$ symmetry and $y^{\prime} \rightarrow-y^{\prime}$ under the $Z_{2}^{\prime}$ symmetry, where $y^{\prime}=y+\pi R / 2$. The physical region in $y$ is given by the interval $[0, \pi R / 2]$. We take the same particle content as the previous section. All gauge bosons and scalar fields are assumed to propagate in the bulk, whilst the matter fields are confined to a four dimensional wall at the orbifold fixed point $y=0$. The bulk gauge group $\mathcal{G}_{q l}$ is reduced by the orbifold compactification. The five dimensional Lagrangian is invariant under the discrete $Z_{2} \times Z_{2}^{\prime}$ symmetry, which acts on the gauge bosons as follows:

$$
\begin{aligned}
W_{\mu}\left(x^{\mu}, y\right) & \rightarrow W_{\mu}\left(x^{\mu},-y\right)=P W_{\mu}\left(x^{\mu}, y\right) P^{-1} \\
W_{5}\left(x^{\mu}, y\right) & \rightarrow W_{5}\left(x^{\mu},-y\right)=-P W_{5}\left(x^{\mu}, y\right) P^{-1} \\
W_{\mu}\left(x^{\mu}, y^{\prime}\right) & \rightarrow W_{\mu}\left(x^{\mu},-y^{\prime}\right)=P^{\prime} W_{\mu}\left(x^{\mu}, y^{\prime}\right) P^{\prime-1} \\
W_{5}\left(x^{\mu}, y^{\prime}\right) & \rightarrow W_{5}\left(x^{\mu},-y^{\prime}\right)=-P^{\prime} W_{5}\left(x^{\mu}, y^{\prime}\right) P^{\prime-1} .
\end{aligned}
$$

We take $P$ and $P^{\prime}$ to be trivial for the $S U_{q}(3), S U_{L}(2)$ and $U_{X}(1)$ gauge bosons. For the $S U_{l}(3)$ gauge bosons we choose $P=\operatorname{diag}(1,1,1)$ and $P^{\prime}=\operatorname{diag}(-1,1,1)$. We write the five dimensional $S U_{l}(3)$ gauge bosons as

$$
\begin{aligned}
G_{l} & =T_{a} G_{l}^{a} \\
& =\left(\begin{array}{ccc}
-\frac{2}{\sqrt{3}} G^{8} & \sqrt{2} Y^{1} & \sqrt{2} Y^{2} \\
\sqrt{2} Y^{1 \dagger} & G^{3}+\frac{1}{\sqrt{3}} G^{8} & \sqrt{2} \tilde{G} \\
\sqrt{2} Y^{2 \dagger} & \sqrt{2} \tilde{G}^{\dagger} & -G^{3}+\frac{1}{\sqrt{3}} G^{8}
\end{array}\right)
\end{aligned}
$$

and find their $Z_{2} \times Z_{2}^{\prime}$ parities to be

$$
\begin{aligned}
& Y_{\mu}^{1}, Y_{\mu}^{2}, Y_{\mu}^{1 \dagger}, Y_{\mu}^{2 \dagger} \rightarrow(+,-), \\
& Y_{5}^{1}, Y_{5}^{2}, Y_{5}^{1 \dagger}, Y_{5}^{2 \dagger} \rightarrow(-,+), \\
& G_{\mu}^{8}, G_{\mu}^{3}, \tilde{G}_{\mu}, \tilde{G}_{\mu}^{\dagger} \rightarrow(+,+), \\
& G_{5}^{8}, G_{5}^{3}, \tilde{G}_{5}, \tilde{G}_{5}^{\dagger} \rightarrow(-,-) .
\end{aligned}
$$

The compact fifth dimension allows one to expand the gauge bosons as a Fourier series, with the $Z_{2} \times Z_{2}^{\prime}$ parities constraining the series as usual.

$$
\begin{aligned}
& \psi_{(+,+)}\left(x^{\mu}, y\right)=\frac{2}{\sqrt{\pi R}}\left(\frac{1}{\sqrt{2}} \psi_{(+,+)}^{(0)}\left(x^{\mu}\right)+\sum_{n=1}^{\infty} \psi_{(+,+)}^{(n)}\left(x^{\mu}\right) \cos \frac{2 n y}{R}\right) \\
& \psi_{(+,-)}\left(x^{\mu}, y\right)=\frac{2}{\sqrt{\pi R}} \sum_{n=1}^{\infty} \psi_{(+,-)}^{(n)}\left(x^{\mu}\right) \cos \frac{(2 n-1) y}{R}, \\
& \psi_{(-,+)}\left(x^{\mu}, y\right)=\frac{2}{\sqrt{\pi R}} \sum_{n=1}^{\infty} \psi_{(-,+)}^{(n)}\left(x^{\mu}\right) \sin \frac{(2 n-1) y}{R}, \\
& \psi_{(-,-)}\left(x^{\mu}, y\right)=\frac{2}{\sqrt{\pi R}} \sum_{n=1}^{\infty} \psi_{(-,-)}^{(n)}\left(x^{\mu}\right) \sin \frac{2 n y}{R}
\end{aligned}
$$

where $\psi$ represents a generic field. Thus the four dimensional charge $1 / 2$ bosons $Y_{\mu}^{1}, Y_{\mu}^{2}, Y_{\mu}^{1 \dagger}$ and $Y_{\mu}^{2 \dagger}$ do not possess zero modes, with the $n$th mode possessing a mass of $(2 n-1) / R$. The fields $G_{\mu}^{8}, G_{\mu}^{3}, \tilde{G}_{\mu}$ and $\tilde{G}_{\mu}^{\dagger}$ all have zero modes, with the higher modes possessing a mass of $2 n / R$. We see that the bulk symmetry $S U_{l}(3)$ has been reduced to $S U_{l}(2) \otimes U_{X^{\prime}}(1)$ at the zero mode level. This is analogous to the $S U(3)$ symmetry reduction employed in [42, 49]. After compactification the zero mode gauge group is

$$
S U_{l}(2) \otimes S U_{q}(3) \otimes S U_{L}(2) \otimes U_{X^{\prime}}(1) \otimes U_{X}(1)
$$

and the next stage of symmetry breaking requires

$$
U_{X^{\prime}}(1) \otimes U_{X}(1) \rightarrow U_{Y}(1),
$$

which shall be achieved by the usual Higgs mechanism. The $Z_{2} \times Z_{2}^{\prime}$ parities of the $\phi_{1,2}$ are

$$
\begin{aligned}
\phi_{1,2}\left(x^{\mu}, y\right) & \rightarrow \phi_{1,2}\left(x^{\mu},-y\right)=P \phi_{1,2}\left(x^{\mu}, y\right), \\
\phi_{1,2}\left(x^{\mu}, y^{\prime}\right) & \rightarrow \phi_{1,2}\left(x^{\mu},-y^{\prime}\right)=P^{\prime} \phi_{1,2}\left(x^{\mu}, y^{\prime}\right),
\end{aligned}
$$

where $P=P^{\prime}=\operatorname{diag}(1,1)$. For $\chi$ we have

$$
\begin{aligned}
\chi\left(x^{\mu}, y\right) & \rightarrow \chi\left(x^{\mu},-y\right)=P \chi\left(x^{\mu}, y\right), \\
\chi\left(x^{\mu}, y^{\prime}\right) & \rightarrow \chi\left(x^{\mu},-y^{\prime}\right)=-P^{\prime} \chi\left(x^{\mu}, y^{\prime}\right),
\end{aligned}
$$


with $P=\operatorname{diag}(1,1,1)$ and $P^{\prime}=\operatorname{diag}(-1,1,1)$, whilst for $\chi^{\prime}$ we take

$$
\begin{gathered}
\chi^{\prime}\left(x^{\mu}, y\right) \rightarrow \chi^{\prime}\left(x^{\mu},-y\right)=-P \chi^{\prime}\left(x^{\mu}, y\right), \\
\chi^{\prime}\left(x^{\mu}, y^{\prime}\right) \rightarrow \chi^{\prime}\left(x^{\mu},-y^{\prime}\right)=P^{\prime} \chi^{\prime}\left(x^{\mu}, y^{\prime}\right),
\end{gathered}
$$

with $P$ and $P^{\prime}$ trivial. Thus $\chi^{\prime} \rightarrow(-,+)$ and vanishes at the $y=0$ boundary of the extra dimension. Under the symmetry reduction

$$
S U_{l}(3) \rightarrow S U_{l}(2) \otimes U_{X^{\prime}}(1),
$$

one has

$$
\chi \rightarrow \chi_{2} \oplus \chi_{1}
$$

where $\chi_{2} \sim(2,1)$ and $\chi_{1} \sim(1,-2)$ have the $Z_{2} \times Z_{2}^{\prime}$ parities

$$
\chi_{1} \rightarrow(+,+) \quad, \quad \chi_{2} \rightarrow(+,-) .
$$

The zero mode for $\chi_{1}$ may develop a VEV and break the gauge symmetry as follows:

$$
S U_{l}(2) \otimes S U_{q}(3) \otimes S U_{L}(2) \otimes U_{X^{\prime}}(1) \otimes U_{X}(1) \rightarrow S U_{l}(2) \otimes S U_{q}(3) \otimes S U_{L}(2) \otimes U_{Y}(1) .
$$

The final stage of symmetry breaking occurs when the neutral components of $\phi_{1,2}$ develop the VEV's $u_{1,2}$ to give

$$
\begin{aligned}
S U_{l}(2) \otimes & S U_{q}(3) \otimes S U_{L}(2) \otimes U_{Y}(1) \\
& \downarrow \\
S U_{l}(2) \otimes & S U_{q}(3) \otimes U_{Q}(1) .
\end{aligned}
$$

\section{GAUGE BOSONS AND EXOTIC LEPTONS}

In the previous section we have shown how a combination of orbifold compactification and the Higgs mechanism may be used to break a five dimensional QL symmetric model down to something resembling the SM. In this section we shall discuss the mass scale of the gauge bosons in the model outlined. We denote the VEV's of the scalars as

$$
\begin{aligned}
\left\langle\chi_{1}\right\rangle & =w \sqrt{2 / \pi R}, \\
\left\langle\phi_{1,2}^{0}\right\rangle & =u_{1,2} \sqrt{2 / \pi R}
\end{aligned}
$$

and we also define $u^{2}=u_{1}^{2}+u_{2}^{2}$. In the basis $\left(W^{0(n)}, C^{(n)}, G^{8(n)}\right)$ the mass squared matrix for the neutral gauge bosons is

$$
\mathcal{M}_{\text {neutral }}^{2(n)}=\left(\begin{array}{ccc}
g_{L}^{2} \frac{u^{2}}{2}+\left(\frac{2 n}{R}\right)^{2} & -g_{X} g_{L} \frac{u^{2}}{2} & 0 \\
-g_{X} g_{L} \frac{u^{2}}{2} & g_{X}^{2} \frac{u^{2}}{2}+\frac{2}{9} g_{X}^{2} w^{2}+\left(\frac{2 n}{R}\right)^{2} & \frac{2 \sqrt{3}}{9} g_{X} g_{S} w^{2} \\
0 & \frac{2 \sqrt{3}}{9} g_{X} g_{S} w^{2} & \frac{2}{3} g_{S}^{2} w^{2}+\left(\frac{2 n}{R}\right)^{2}
\end{array}\right)
$$

where $g_{L}\left[g_{X}\right]$ is the $S U_{L}(2)\left[U_{X}(1)\right]$ coupling constant and $g_{S}$ denotes the common $S U_{l}(3)$ and $S U_{q}(3)$ coupling constant. Note that these are dimensionless constants, related to the dimensionfull five dimensional Lagrangian constants via $g_{S}=g_{S}^{5} \sqrt{2 / \pi R}$ etc. Assuming $g_{S}^{2} \gg g_{X}^{2}, g_{L}^{2}$ one may write the eigenvalues as

$$
\begin{aligned}
M_{\gamma}^{2(n)} & =\left(\frac{2 n}{R}\right)^{2}, \\
M_{Z}^{2(n)} & \simeq \frac{1}{2}\left(g_{X}^{2}+g_{L}^{2}\right) u^{2}-\frac{g_{X}^{2}}{6 g_{S}^{2}} u^{2}+\left(\frac{2 n}{R}\right)^{2}, \\
M_{Z^{\prime}}^{2(n)} & \simeq \frac{2}{3} g_{S}^{2} w^{2}\left\{1+\frac{g_{X}^{2}}{3 g_{S}^{2}}\right\}+\left(\frac{2 n}{R}\right)^{2} .
\end{aligned}
$$

Note that the zero modes possess the same eigenvalues as the neutral gauge bosons in the minimal four dimensional QL symmetric model [1, 3]. In fact these zero modes couple to fermions in exactly the same way as the neutral gauge bosons in the minimal QL symmetric model, making the phenomenology of these sates identical to that of the neutral gauge bosons studied in [3].

The zero modes consist of the massless photon, the $Z$ boson with mass of order $u$, the electroweak scale, and an additional neutral boson $Z^{\prime}$ with mass of order $w$, the $U_{X^{\prime}}(1) \otimes U_{X}(1)$ symmetry breaking scale. The phenomenological bound of $M_{Z^{\prime}}>720 \mathrm{GeV}$ obtained in [3] also applies to the zero mode $Z^{\prime}$ boson in the present model. Thus we obtain a lower bound on the $U_{X^{\prime}}(1) \otimes U_{X}(1)$ symmetry breaking scale of $w \gtrsim 1 \mathrm{TeV}$, which is low enough to permit observation of the $Z^{\prime}$ boson and the exotic leptons at the LHC. 
The mass of the $W$ bosons in the present model is

$$
M_{W}^{2(n)}=\frac{1}{2} g_{L}^{2} u^{2}+\left(\frac{2 n}{R}\right)^{2},
$$

with the zero mode corresponding to the usual $W$ bosons. The mass of the charged $1 / 2$ bosons is

$$
M_{Y^{1}}^{2(n)}=M_{Y^{2}}^{2(n)}=\frac{1}{2} g_{S}^{2} w^{2}+\left(\frac{2 n-1}{R}\right)^{2},
$$

with the zero mode absent. Thus we see that for $1 / R>w$ the mass of the $Y$ bosons is set by the size of the extra dimensions. In [3] a rough lower bound on the mass of the $Y$ bosons was obtained by considering the rare decay $\mu \rightarrow 3 e$, which is radiatively induced in QL symmetric models due to the presence of the exotic leptons. The result is

$$
M_{Y} \geq(5 \mathrm{TeV}) \times c
$$

where $c$ is an $O(1)$ number which depends on mixing angles and the mass of the exotic leptons. In the present model this translates into the rough bound

$$
\frac{1}{R} \geq 5 \mathrm{TeV} \text {. }
$$

Note that the present model provides a rationale for the hierarchy $M_{Z^{\prime}}<M_{Y}$ with the mass of these bosons originating from different symmetry breaking mechanisms. The $Y$ bosons, possessing no zero mode, acquire mass at the inverse compactification scale $1 / R$, whilst the lightest $Z^{\prime}$ obtains mass at the $U_{X^{\prime}}(1) \otimes U_{X}(1)$ symmetry breaking scale via the Higgs mechanism.

The exotic charge $1 / 2$ leptons, known as liptons in the literature [3], also develop mass at the $U_{X^{\prime}}(1) \otimes U_{X}(1)$ symmetry breaking scale $w$. These states are confined by the unbroken $S U_{l}(2)$ symmetry and form exotic hadrons, an analysis of which may be found in [3] and more recently in [11] (see also [7]). Here we briefly surmise some of the interesting phenomenology associated with these states.

The liptons are confined into two particle bound states by the unbroken $S U_{l}(2)$ symmetry. The bound states formed by the lightest lipton (which we call $L_{1}$ ) can be produced by virtual $W, Z$ and $\gamma$ decays in future colliders such as the LHC. The $S U_{l}(2)$ gauge interactions preserve a global flavour symmetry $S U_{F}(2)$ with $L_{1}$ and its antiparticle $L_{1}^{c}$ forming a doublet representation of $S U_{F}(2)$. The exotic hadron flavour structure can be determined by the group product

$$
2 \otimes 2=1_{A} \oplus 3_{S},
$$

where the subscripts indicate the symmetry properties of the state under interchange of the liptons. The ground states of the exotic hadrons have zero angular momentum and thus the Pauli principle dictates that the flavour triplet will have spin-1 and the flavour singlet will have spin- 0 . These states may be denoted as

$$
\begin{aligned}
\rho^{+} & =L_{1} L_{1}, \quad \rho^{-}=L_{1}^{c} L_{1}^{c}, \\
\rho^{0} & =\frac{1}{\sqrt{2}}\left(L_{1} L_{1}^{c}+L_{1}^{c} L_{1}\right), \\
\xi^{0} & =\frac{1}{\sqrt{2}}\left(L_{1} L_{1}^{c}-L_{1}^{c} L_{1}\right),
\end{aligned}
$$

and all decay into SM particles, with decay modes such as

$$
\begin{aligned}
\rho^{+} & \rightarrow e^{+} \nu, \\
\rho^{0} & \rightarrow e^{+} e^{-}, \\
\xi^{0} & \rightarrow 2 \gamma .
\end{aligned}
$$

Let us now stop to contrast the exotic particle spectrum in our model with that of the minimal QL symmetric model. In the minimal QL symmetric model the exotic bosons and fermions all develop mass at the scale $w$. The slightly more stringent bound on the $Y$ bosons requires this scale to be larger than a $\mathrm{TeV}$ or so. Our five dimensional model decouples the scale of some of these exotic particles. The $Y$ bosons develop mass at the inverse compactification scale due to the absence of zero modes. The additional neutral boson $Z^{\prime}$ and the liptons develop mass at the $U_{X^{\prime}}(1) \otimes U_{X}(1)$ symmetry breaking scale, which is less than the inverse compactification scale. At the scale $M_{Y}$ the first Kaluza-Klein modes of the photon, the $Z$ and the $Z^{\prime}$ also appear, providing a clear distinction between the higher dimensional model and the minimal four dimensional model.

We note that we have considered the minimal Higgs sector to date. Some studies of QL symmetric models have included the additional scalars [1, 3]

$$
\Delta_{l} \sim(\overline{6}, 1,1,-4 / 3), \quad \Delta_{q} \sim(1, \overline{6}, 1,4 / 3),
$$

which form a pair under the discrete QL symmetry. If $\left\langle\Delta_{q}\right\rangle=$ 0 and the component of $\Delta_{l}$ which forms a $(1,4)$ representation of $S U_{l}(2) \otimes U_{X^{\prime}}(1) \subset S U_{l}(3)$ develops a non-zero $\operatorname{VEV}\left\langle\Delta_{l}\right\rangle$, the QL symmetric gauge group is broken down to $S U_{l}(2) \otimes S U_{q}(3) \otimes S U_{L}(2) \otimes U_{Y}(1)$ at the scale $\left\langle\Delta_{l}\right\rangle$. This extension has the advantage of giving a Majorana mass to the right-chiral neutrinos at the scale $\left\langle\Delta_{l}\right\rangle$, allowing one to employ the see-saw mechanism to explain the relative lightness of the neutrinos.

In the minimal QL symmetric model one requires highly tuned Dirac Yukawa couplings to produce very light neutrinos, making the addition of the states $\Delta$ an attractive extension. However this modification couples the mass of the exotic gauge bosons $Y$ and $Z^{\prime}$ to the right-chiral Majorana mass scale, which is typically required to be larger than $10^{11} \mathrm{GeV}$ or so, depending on how small one tolerates the neutrino Dirac mass matrix Yukawa couplings. Thus the exotic bosons become unobservably heavy. The liptons do not develop mass at the scale $\left\langle\Delta_{l}\right\rangle$ and one still requires the field $\chi$ to develop a VEV (though it is not breaking any symmetry) to give mass to these fermions.

Thus in the extended four dimensional QL symmetric model the liptons have masses of order $w$ and the exotic gauge bosons have mass at the scale $\left\langle\Delta_{l}\right\rangle$. Note that the hierarchy $\left\langle\Delta_{l}\right\rangle \gg w$ is still allowed in these models so that the exotic fermions may be observed at $\mathrm{TeV}$ energies. Again this spectrum contrasts vividly with that obtained in the five dimensional model. In the four dimensional model only the liptons become accessible at the scale $w$, whilst the five dimensional model also requires the $Z^{\prime}$ boson to appear at this scale. 


\section{FERMIONS, PROTON DECAY AND NEUTRINO MASS}

In the present work we shall assume that the SM fermions are confined to the brane at $y=0$. We have seen in the previous sections that the mass scale of the $Y$ bosons is set by the inverse compactification scale in our framework, which is bound to be greater than $5 \mathrm{TeV}$. Given that all fermions are assumed to be stuck at the $y=0$ wall, non-renormalizable proton decay inducing operators will arise and we must ensure that these are adequately suppressed.

In the SM proton decay occurs via the dimension six operator

$$
\frac{h}{\Lambda_{S M}^{2}} \epsilon_{\alpha \beta \gamma} Q^{\alpha} Q^{\beta} Q^{\gamma} L,
$$

where $\alpha, \beta, \gamma=1,2,3$ are colour indices, $\Lambda_{S M}$ is the SM UV cutoff, $Q(L)$ denotes quark (lepton) operators and $h$ is a dimensionless coupling. This operator leads to the decay $p \rightarrow e^{+} \pi^{0}$. Given that experimental bounds require the lifetime of the proton to be in excess of $1.6 \times 10^{33}$ years, one requires $\Lambda_{S M} \sim 10^{16} \mathrm{GeV}$ [50]. In a four dimensional QL symmetric framework the lowest dimension non-renormalizable operator that induces proton decay has dimension seven,

$$
\frac{h}{\Lambda_{q l}^{3}} \epsilon_{\alpha \beta \gamma} Q^{\alpha} Q^{\beta} Q^{\gamma} \chi_{\bar{\alpha}}^{\dagger} L^{\bar{\alpha}},
$$

where $\bar{\alpha}=1,2,3$ is the leptonic colour index and $\Lambda_{q l}$ is the UV cutoff. When $\chi$ develops a VEV this leads to an effective operator of the form (33). Relating the two cutoff's gives

$$
\Lambda_{q l}=\Lambda_{S M}^{2 / 3}\langle\chi\rangle^{1 / 3}
$$

and using the order TeV lower bound on $\langle\chi\rangle$ leads to

$$
\Lambda_{q l} \geq 5 \times 10^{11} \mathrm{GeV}
$$

If quarks and leptons are localized at $y=0$ in our five dimensional QL model the lowest dimension nonrenormalizable operator which leads to proton decay is the equivalent of (34):

$$
\frac{h}{\Lambda_{q l}^{7 / 2}} \epsilon_{\alpha \beta \gamma} Q^{\alpha} Q^{\beta} Q^{\gamma} \chi_{\bar{\alpha}}^{\dagger} L^{\bar{\alpha}},
$$

where $\chi$ is now a five dimensional field. If we assume that the cut-off is only a couple of orders of magnitude larger than the inverse compactification scale, say $\Lambda_{q l} \sim 100 / R$, we arrive at the bound

$$
\Lambda_{q l} \geq 2 \times 10^{11} \mathrm{GeV}
$$

which is incompatible with a low fundamental scale. The bound on $\Lambda_{q l}$ corresponds to an inverse compactification scale of order $10^{9} \mathrm{GeV}$ and consequently the phenomenology associated with the $Y$ bosons will not appear at low energies within in our framework. The bounds from the proton decay rate do not disturb the bounds on the $Z^{\prime}$ boson mass obtained earlier and this boson can, in principle, still appear at order $\mathrm{TeV}$ energies.

As mentioned in the previous section, it is known that in a four dimensional QL symmetric model one may suppress the known neutrino masses relative to the electroweak scale by introducing scalars forming a six dimensional representation of $S U_{l}(3)$ (and their QL symmetry partners). However this forces the $Z^{\prime}$ mass up to the right-chiral neutrino Majorana mass scale. In this section we shall employ a mechanism previously employed in QL symmetric models [6] to allow the neutrinos to acquire masses suppressed relative to the electroweak scale (see [51, 52] for earlier implementations of this mechanism). The mechanism does not require the introduction of any additional high energy scales and allows the zero mode $Z^{\prime}$ boson to retain an order TeV mass.

We add three fermions $S_{L} \sim(1,1,1,0)$ to the particle spectrum, which transform trivially under the discrete QL symmetry. These fermions are also assumed to be stuck to the wall at $y=0$. The gauge symmetries allow the additional Lagrangian terms

$$
\mathcal{L}_{S_{L}}=h_{3}^{5}\left[\bar{S}_{L} \chi^{\dagger} N_{R}+\bar{S}_{L} \chi^{\prime \dagger} d_{R}\right]+M_{S} \overline{S_{L}^{c}} S_{L}+\text { H.c. },
$$

where we omit the delta function indicating that the fermions are localized at the $y=0$ brane.

After symmetry breaking is complete the non zero VEV's for $\phi_{1,2}$ and $\chi$ result in the neutrino mass matrix

$$
\mathcal{M}_{\nu}=\left(\begin{array}{ccc}
0 & m & 0 \\
m & 0 & M \\
0 & M & M_{S}
\end{array}\right),
$$

in the Majorana basis $\left(\nu_{L},\left(\nu_{R}\right)^{c}, S_{L}\right)$, where for simplicity we show only one generation. Here $\nu_{L}\left(\nu_{R}\right)$ is a normal electroweak doublet (singlet) neutrino whilst

$$
m=\lambda_{1} u_{2}+\lambda_{1}^{\prime} u_{1}^{*} \sim u_{1,2} \text { and } M=h_{3} w,
$$

where $h_{3}=h_{3}^{5} \sqrt{2 / \pi R}$. In the limit $M_{S} \rightarrow 0$ the mass eigenstates consist of one massless Weyl neutrino

$$
\nu_{W L}=\cos \theta \nu_{L}-\sin \theta S_{L}
$$

and one Dirac neutrino with mass $\sqrt{m^{2}+M^{2}}$,

$$
\begin{aligned}
& \nu_{D L}=\sin \theta \nu_{L}+\cos \theta S_{L}, \\
& \nu_{D R}=\nu_{R},
\end{aligned}
$$

where $\tan \theta=m / M$. When $M_{S}$ is turned on, and the hierarchy $M_{S} \ll m \ll M$ exists, the massless state develops a mass $M_{S} m^{2} / M^{2}$ and the Dirac neutrino becomes two Majorana neutrinos with mass splitting of order $M_{S}$. Note that the light state consists predominantly of the normal electroweak neutrino $\nu_{L}$ under the hierarchy $m \ll M$. If one takes $M \sim 1 \mathrm{TeV}$, in line with the lower bound on the $Z^{\prime}$ boson masses, and $m \leq 10 \mathrm{GeV}$, then $\theta \leq 0.6^{\circ}$. Requiring $M_{S} m^{2} / M^{2} \sim 0.1 \mathrm{eV}$ to accommodate solar and atmospheric neutrino oscillation data then gives $M_{S} \sim 1 \mathrm{keV}$. As noted in [6], the hierarchy between the scale $M_{S}$ and $u_{1,2}, w$ is perturbatively stable due to the enhanced symmetry of the theory in the limit $M_{S} \rightarrow 0$. 
The three generation case is readily obtained by converting $m, M$ and $M_{S}$ to $3 \times 3$ matrices in flavour space. The result been three mixed, light neutrinos, with mass of order $M_{s} m^{2} / M^{2}$, which predominantly contain the usual electroweak neutrinos $\nu_{e L}, \nu_{\mu L}$ and $\nu_{\tau L}$.

Note that in a four dimensional QL model the Lagrangian term $S_{L} \chi^{\prime} d$ in eq. (39) breaks baryon number and allows the proton to decay. However in our five dimensional model the $Z_{2} \times Z_{2}^{\prime}$ parities for $\chi^{\prime}$ ensure that it vanishes at the $y=0$ brane. Thus after integrating over the extra dimension this term disappears from the four dimensional theory and baryon number is conserved. Consequently proton decay may only be mediated by heavy fields in the more fundamental theory.

This mechanism of obtaining light neutrino masses is particularly well suited to higher dimensional theories when the right-chiral neutrinos form non-trivial representations of some additional gauge symmetry. Provided the additional symmetry is broken above the electroweak scale one may obtain light neutrinos with only a mild hierarchy between the electroweak scale and the additional symmetry breaking scale. Whilst we have used this mechanism in a QL symmetric model it could also be applied in, $e g$ a higher dimensional left-right symmetric framework (examples of which include [39, 40, 41]).

We note that it may be possible to construct a five dimensional QL symmetric model compatible with a low fundamental scale by allowing the fermions to propagate in the bulk. One could then localize quarks and leptons at different points in the extra dimension [53], thereby reducing their fifth dimensional wavefunction overlaps and suppressing the proton decay rate. This idea is currently under investigation [54]

\section{CONCLUSION}

We have studied the five dimensional QL symmetric gauge group. The $S U_{l}(3)$ symmetry was reduced to $S U_{l}(2) \otimes$ $U_{X^{\prime}}(1)$ as a result of the compactification of the fifth dimension. The charged 1/2 Y bosons, corresponding to the bro- ken $S U_{l}(3)$ generators, form a Kaluza-Klein tower with no zero mode. Consequently the lightest $Y$ bosons possesses a mass of order $1 / R$, with direct bounds giving $1 / R \geq 5 \mathrm{TeV}$. However the prevention of rapid proton decay requires a UV cut-off of order $10^{11} \mathrm{GeV}$. If one assumes that the more fundamental theory takes over just beyond the inverse compactification scale one expects $1 R \gtrsim 10^{9} \mathrm{GeV}$, and the $Y$ bosons disappear from the low energy spectrum.

The subsequent breaking of $U_{X^{\prime}}(1) \otimes U_{X}(1)$ by the Higgs mechanism produces a massive neutral gauge boson at a scale phenomenologically required to be greater than $1 \mathrm{TeV}$. The liptons also acquire mass at this stage of symmetry breaking. The photon, $Z$ and $W$ bosons form part of a Kaluza Klein tower, with the $n=0$ modes corresponding to the usual SM gauge bosons. The bounds on the exotic states are low enough for the zero mode of the additional neutral gauge boson to appear at an $e^{+} e^{-}$collider operating at $\mathrm{TeV}$ energies and for the exotic leptons to appear at the LHC.

This is to be contrasted with the four dimensional QL symmetric model, where the massive gauge bosons not contained within the SM acquire mass at a common symmetry breaking scale. This scale is required to be of order $10^{11} \mathrm{GeV}$ or larger if the see-saw mechanism is employed, rendering the exotic gauge bosons unobservable at low energies.

We have also shown that one may obtain light neutrinos within the five dimensional framework without introducing any additional high energy scales, and although our model is not compatible with a low fundamental scale this situation may change if the fermions are assumed to propagate in the bulk [54].

\section{Acknowledgements}

KM thanks T. Gherghetta, R. Volkas, A. Demaria, D. George, A. Coulthurst and R. Foot for clarifying communications. This work was supported in part by the Australian Research Council.
[1] R. Foot and H. Lew, Phys. Rev. D 41 (1990) 3502.

[2] R. Foot and H. Lew, Phys. Rev. D 42 (1990) 945.

[3] R. Foot, H. Lew and R. R. Volkas, Phys. Rev. D 44, 1531 (1991).

[4] Y. Levin and R. R. Volkas, Phys. Rev. D 48, 5342 (1993) |arXiv:hep-ph/9308256.

[5] D. S. Shaw and R. R. Volkas, Phys. Rev. D 51, 6490 (1995) |arXiv:hep-ph/9410350|.

[6] R. Foot and R. R. Volkas, Phys. Lett. B 358, 318 (1995) |arXiv:hep-ph/9505331|.

[7] K. S. Babu, E. Ma and S. Willenbrock, Phys. Rev. D 69, 051301 (2004) arXiv:hep-ph/0307380|.

[8] S. L. Chen and E. Ma, Mod. Phys. Lett. A 19, 1267 (2004) |arXiv:hep-ph/0403105|.

[9] A. Demaria, C. I. Low and R. R. Volkas, Phys. Rev. D 72, 075007 (2005) [Erratum-ibid. D 73, 079902 (2006)] arXiv:hep-ph/0508160.

[10] A. Demaria, C. I. Low and R. R. Volkas,
arXiv:hep-ph/0603152

[11] R. Foot and R. R. Volkas, arXiv:hep-ph/0607047

[12] J. Scherk and J. H. Schwarz, Phys. Lett. B 82, 60 (1979).

[13] J. Scherk and J. H. Schwarz, Nucl. Phys. B 153, 61 (1979).

[14] Y. Hosotani, Phys. Lett. B 126, 309 (1983).

[15] P. Candelas, G. T. Horowitz, A. Strominger and E. Witten, Nucl. Phys. B 258, 46 (1985).

[16] E. Witten, Nucl. Phys. B 258, 75 (1985).

[17] L. J. Dixon, J. A. Harvey, C. Vafa and E. Witten, Nucl. Phys. B 261, 678 (1985).

[18] Y. Kawamura, Prog. Theor. Phys. 103, 613 (2000) |arXiv:hep-ph/9902423|.

[19] Y. Kawamura, Prog. Theor. Phys. 105, 999 (2001) |arXiv:hep-ph/0012125|.

[20] Y. Kawamura, Prog. Theor. Phys. 105, 691 (2001) |arXiv:hep-ph/0012352|.

[21] G. Altarelli and F. Feruglio, Phys. Lett. B 511, 257 (2001) |arXiv:hep-ph/0102301|. 
[22] A. B. Kobakhidze, Phys. Lett. B 514, 131 (2001) |arXiv:hep-ph/0102323|.

[23] L. J. Hall and Y. Nomura, Phys. Rev. D 64, 055003 (2001) |arXiv:hep-ph/0103125|.

[24] L. J. Hall and Y. Nomura, Phys. Rev. D 66, 075004 (2002) |arXiv:hep-ph/0205067|.

[25] A. Hebecker and J. March-Russell, Nucl. Phys. B 613, 3 (2001) |arXiv:hep-ph/0106166|.

[26] W. F. Chang and J. N. Ng, JHEP 0310, 036 (2003) |arXiv:hep-ph/0308187|.

[27] A. Hebecker and J. March-Russell, Nucl. Phys. B 625, 128 (2002) |arXiv:hep-ph/0107039|.

[28] C. Csaki, G. D. Kribs and J. Terning, Phys. Rev. D 65, 015004 (2002) arXiv:hep-ph/0107266|.

[29] N. Maru, Phys. Lett. B 522, 117 (2001) |arXiv:hep-ph/0108002|.

[30] L. J. Hall, Y. Nomura, T. Okui and D. R. Smith, Phys. Rev. D 65, 035008 (2002) |arXiv:hep-ph/0108071|.

[31] R. Dermisek and A. Mafi, Phys. Rev. D 65, 055002 (2002) |arXiv:hep-ph/0108139|.

[32] T. Watari and T. Yanagida, Phys. Lett. B 519, 164 (2001) |arXiv:hep-ph/0108152|.

[33] K. S. Babu, S. M. Barr and B. Kyae, Phys. Rev. D 65, 115008 (2002) |arXiv:hep-ph/0202178|.

[34] F. Paccetti Correia, M. G. Schmidt and Z. Tavartkiladze, Nucl. Phys. B 649, 39 (2003) |arXiv:hep-ph/0204080|.

[35] F. Paccetti Correia, M. G. Schmidt and Z. Tavartkiladze, Phys. Lett. B 545, 153 (2002) |arXiv:hep-ph/0206307|.

[36] N. Haba and Y. Shimizu, arXiv:hep-ph/0210146

[37] S. M. Barr and I. Dorsner, Phys. Rev. D 66, 065013 (2002) |arXiv:hep-ph/0205088|.

[38] I. Dorsner, Phys. Rev. D 69, $056003 \quad$ (2004)
arXiv:hep-ph/0310175|.

[39] Y. Mimura and S. Nandi, Phys. Lett. B 538, 406 (2002) |arXiv:hep-ph/0203126|.

[40] R. N. Mohapatra and A. Perez-Lorenzana, Phys. Rev. D 66, 035005 (2002) |arXiv:hep-ph/0205347|.

[41] G. Perez, Phys. Rev. D 67, 013009 (2003) |arXiv:hep-ph/0208102|.

[42] I. Gogoladze, Y. Mimura and S. Nandi, Phys. Lett. B 554, 81 (2003) arXiv:hep-ph/0210320 .

[43] L. J. Hall and Y. Nomura, Phys. Lett. B 532, 111 (2002) |arXiv:hep-ph/0202107|.

[44] T. j. Li and W. Liao, Phys. Lett. B 545, 147 (2002) |arXiv:hep-ph/0202090|.

[45] S. Dimopoulos, D. E. Kaplan and N. Weiner, Phys. Lett. B 534, 124 (2002) |arXiv:hep-ph/0202136|.

[46] C. H. Chang, W. F. Chang and J. N. Ng, Phys. Lett. B 558, 92 (2003) |arXiv:hep-ph/0301271|.

[47] W. F. Chang and J. N. Ng, Phys. Rev. D 69, 056005 (2004) |arXiv:hep-ph/0312199|.

[48] C. D. Carone and J. M. Conroy, Phys. Rev. D 70, 075013 (2004) |arXiv:hep-ph/0407116.

[49] C. D. Carone and J. M. Conroy, Phys. Lett. B 626, 195 (2005) |arXiv:hep-ph/0507292|.

[50] P. Nath and P. F. Perez, arXiv:hep-ph/0601023

[51] D. Wyler and L. Wolfenstein, Nucl. Phys. B 218, 205 (1983).

[52] R. N. Mohapatra and J. W. F. Valle, Phys. Lett. B 177 (1986) 47.

[53] N. Arkani-Hamed and M. Schmaltz, Phys. Rev. D 61, 033005 (2000) arXiv:hep-ph/9903417.

[54] K.L. McDonald and B. H. J. McKellar, in preparation. 